Original Research

\title{
Comparing the Perspectives of Professionals and Relatives Toward the Quality of Palliative Care: A Qualitative Study
}

\author{
Emily Reeves ${ }^{1,2,{ }^{*}}$, Brigitte Liebig ${ }^{2,3}$, Eveline Degen Jermann ${ }^{2}$
}

1. University of Basel, Department of Psychology, Missionsstrasse 60/62 4005, Basel, Switzerland; E-Mail: emily.reeves@unibas.ch

2. Fachhochschule Nordwestschweiz FHNW, School of Applied Psychology, Riggenbachstrasse 16, Olten, Switzerland; E-Mails: brigitte.liebig@fhnw.ch; eveline.degen@fhnw.ch

3. University of Basel, Institute for Sociology, Petersgraben 27, 4051 Basel, Switzerland; E-Mail: brigitte.liebig@unibas.ch

* Correspondence: Emily Reeves; E-Mail: emily.reeves@unibas.ch

Academic Editor: Leila Kozak

Special Issue: Integrative Therapies in Palliative Care

OBM Integrative and Complementary Medicine

2020 , volume 5 , issue 4

doi:10.21926/obm.icm.2004041
Received: July 14, 2020

Accepted: September 23, 2020

Published: October 20, 2020

\begin{abstract}
High-quality care is an important aim of palliative care services. However, the quality of care is a complex phenomenon and is subjectively perceived by those who are closest to the services and are often evaluated poorly. Prior research has highlighted the difficulties in evaluating the quality of care because of different perspectives, and still, only a little is understood about the perception of the quality of care in palliative care services. Investigating the perspectives of key individuals involved in palliative care, such as palliative professionals and relatives of palliative patients, can provide invaluable insight to understand the quality of care. To identify the perceptions of palliative professionals and relatives of the patients toward the quality of palliative care along with identifying the similarities and divergences in the perceptions, as well as highlighting the complexity of quality in palliative care settings while indicating their areas of improvement. This was a qualitative study that involved semistructured interviews for the evaluation of palliative care guided by the proposed system
\end{abstract}

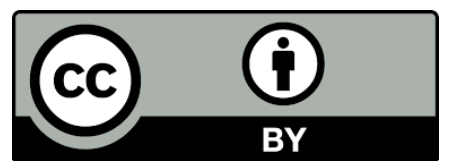

(c) 2020 by the author. This is an open access article distributed under the conditions of the Creative Commons by Attribution License, which permits unrestricted use, distribution, and reproduction in any medium or format, provided the original work is correctly cited. 
approach model [1]. The interviews were conducted with a total number of 38 professionals working in palliative care along with 26 relatives of palliative patients across four Swiss cantons (Ticino, Basel, Vaud, and Lucerne). These professionals worked in primary palliative care (PPC) and specialized palliative care (SPC), where 11 general practitioners (GP's) and 12 nurses were from primary palliative care services. Additionally, 15 interviews were conducted with 9 specialized medical doctors (e.g., oncologists) and 6 specialized palliative care nurses from different hospitals, hospices, mobile palliative care teams (MPCT's), and specialist private practice. The results revealed considerable differences between the perspectives of professionals and relatives regarding the following aspects of care: 1) the availability, 2) clientcenteredness (including respect for patient's wishes and relative's involvement in care, 3) satisfaction (including physical and psychosocial), and 4) communication and information transfer. Mainly, the family members felt that care was not being always readily available and coordinated poorly, the patient's needs were overlooked because of the lack of time. Also, some professionals were perceived to be incompetent with a lack of communication and negligence toward the psychosocial needs of the patient. On the other hand, professionals indicated that relatives may be too involved in the patient care and might have expressed concern due to the high risk of burn out caused by extreme stress and emotionally taxing circumstances of palliative care. The different perspectives uncovered both the similarities and discrepancies, representing a demand for addressing the shortcomings of palliative care services. Specifically, findings indicated that professionals may overestimate the quality of palliative care. Also, future initiatives were needed to ensure that the family members of palliative patients were adequately supported, both psycho-socially and financially, justifying the investment. These findings would help in informing the health policy about the aspects of care that require specific attention to improve the overall quality of care and urge future research to explore the best ways to support these aspects of care.

\section{Keywords}

Palliative home care; quality of care; comparing perspectives; family caregivers; palliative professionals

\section{Introduction}

High-quality care is a vital goal for palliative care services [2], not only because the palliative patients who often have critical demand for effective care services for both pain and symptom management but also to provide good physical and psychosocial support functions [3]. However, quality of care is a complex phenomenon, not necessarily determined by the outcomes of care but constitutes multiple factors and is evaluated subjectively by the individuals closest to care, i.e., the palliative professionals and the relatives of palliative patients [4]. Moreover, ensuring the efficient delivery of care is not always guaranteed and the standard of providing palliative care service is often deemed unsatisfactory [5]. Yet, only little is understood about the perception of the quality of care in palliative services. Thus, the present study aims to explore perceptions of the quality of palliative care, as well as highlight the complexity of the phenomenon. Specifically, the objectives 
are to identify similarities and differences in the perspectives regarding the key elements of the quality of palliative care and also identify the avenues to refine their existing indicators. These are important aims that ensure the clinicians in palliative care settings are well informed about the experiences and perspectives of the patients' relatives, allowing them to potentially adapt and implement the interventions accordingly to serve the patients better and also those who care for them $[4,6]$. Thus, this qualitative study is based on the data concerning the perceptions of quality of palliative care derived from health care professionals working in primary and specialized palliative care settings, as well as the relatives of palliative patients. Their insights are crucial for informing the policy-makers, healthcare managers, and personnel in the sphere of palliative care to identify the potential areas of improvement and promote high-quality of care provision. The following section provides a background to the quality of care specific to the context of palliative care services, including a description of the 'proposed system approach model for evaluating the quality of palliative care' [1], which is the basis of this investigation along with a description about the value of exploring perceptions of palliative professionals and relatives of the patients to understand the quality of palliative care services. Subsequently, the methodology of this qualitative study and its findings are described with the discussion of the findings, followed by the conclusion, recommendations for future research, and limitations of the study.

\subsection{Quality of Palliative Care}

Based on several descriptions in the literature, the World Health Organization (WHO) defines the quality of care as "the extent of health care services provided to individuals and patient population to improve the desired health outcomes" and proposes that to achieve this; the health care must be safe, effective, timely, efficient, equitable, and people-centric [7]. Specifically, the quality of care is considered to be of a high standard if the preferences of patients and relatives are taken into account. Also, their perception of care constitutes the dimensions of quality of care [6]. A highly recognized model for the evaluation of palliative care was developed by Bainbridge and colleagues [1]. According to the 'proposed system approach for the evaluation of palliative care', the following factors are indicative of the quality of care: the availability (i.e., palliative services being accessible to those who need them, in time), the client centeredness (i.e., respect for patient's wishes and adequate involvement of relatives of patients in care), satisfaction (including physical and psychosocial care), and communication and information transfer (i.e., collaboration and transfer of patient information between the palliative care providers). Moreover, for palliative care services to be evaluated positively, it is ought to follow the above-mentioned factors, and only then it can be considered as high standard (i.e., patients and relatives should have frequent, clear communication about the care provided).

\subsection{Perceptions of Quality of Care}

The perspectives of individuals close to palliative patients are imperative for understanding the quality of care in palliative care services [8]. Naturally, the health care professionals (e.g., general practitioners (GP's), nurses, specialized doctors, such as oncologists and specialized nurses), and palliative patients with their relatives, were investigated to understand the quality of care, considering that the extensive experience would make their insights invaluable. However, the perspectives of relatives of palliative patients are more relevant, not only because of their closeness 
to the patient but also because of their increasing involvement in palliative care services [9]. A growing body of research supports that relatives are increasingly being embedded in the palliative care teams, particularly as more aspects of palliative care are provided in the family home environment of the patients [10] and many relatives are receiving palliative training [11] as well to assume the role of primary caregivers, i.e., individuals who provide support without payment [12]. Moreover, while recruiting patients, their critical health status makes it challenging to obtain their feedback for studies to investigate the quality of palliative care [2]. Hence, the insight of those who are closest to the patients is crucial, if not at least a proxy to the perception of patients is needed [13]. Moreover, since palliative care services seek to support not only the patient but also their close relatives [14], their experience of the quality of care also becomes crucial. Yet, research that has compared the perspectives of different people related to care has often identified discrepancies in the perspectives of the quality of care $[8,15,16]$. For example, professionals were shown to overestimate the quality of care delivery to patients and relatives [17]. Identifying the perspectives of quality of care in palliative services allows us to gain a broader, more accurate picture of the quality of care and identify the areas of improvement. However, a majority of the existing literature has investigated perceptions of specific aspects of quality of care, such as perceived patientcenteredness [18] or availability of support services from the relatives [19]. Similarly, in Switzerland, some studies have explored the factors that might indicate the quality of care in palliative settings, such as coordination of care [20] or interprofessional collaboration within the palliative care teams [21]. However, there is a limited understanding of how the quality of care in palliative care services should be perceived. Owing to the gap in research, this article explored the views of the quality of care in palliative services from the perspectives of those who are closest to this field, i.e., palliative professionals and relatives of the patients. Specifically, this study compares their perceptions to bring inconsistencies and similarities to light and to acquire a well-informed understanding of the quality of care in palliative services, according to those closest to the field.

\section{Methods}

The qualitative study design was adopted to explore the perspectives of quality of care from palliative professionals and relatives of palliative patients in Switzerland. Results were based on semi-structured interviews with a total of 38 professionals working in palliative care and 26 relatives of palliative patients. The professionals were from primary palliative care (PPC) and specialized palliative care (SPC), including 11 general practitioners (GP's) and 12 nurses from primary palliative care services, as well as 15 interviews conducted with 9 specialized medical doctors (e.g., oncologists) and 6 specialized palliative care nurses working in different hospitals, hospices, mobile palliative care teams (MPCT's), and specialist private practice. Also, a total of 26 family members of palliative patients participated in 22 interviews and one group interview (involving three daughters and one son of a palliative care patient). The interviews were conducted in different regions/cantons of Switzerland, namely Basel-City, Lucerne, Ticino, and Vaud. These cantons were selected to accurately reflect the rural and urban regions of Switzerland with differently developed policy contexts for palliative care. 
Table 1 Demographic data of the interviewees

\begin{tabular}{llllll}
\hline Participants & Number & Gender & Age (Mean) & Age (Mean) overall \\
& & Female & Male & & \\
\hline GP's (PPC) & 11 & 4 & 8 & 56.6 & 52.3 \\
Nurses (PPC) & 12 & 12 & 0 & 48.1 & \\
GP's (SPC) & 9 & 8 & 4 & 48 & 50.6 \\
Nurses (SPC) & 6 & 3 & 1 & 57.7 & $60(59.7)$ \\
Family members & 26 & 19 & 7 & & \\
\hline
\end{tabular}

\subsection{Data Collection}

The data for the study were collected between January 2018 and October 2018. In each canton, the participants were identified via a personal referral (i.e., word of mouth) from the professionals who were working in palliative care and were already known to the researchers through prior collaboration, either recruited via email invitation or telephone call. The participants included in the study were interviewed by three researchers in a semi-structured interview format and in a language that corresponded to the official language of the cantons (Vaud: French, Ticino: Italian, or Lucerne/Basel: German). Interviews lasted for $60 \mathrm{~min}$ on an average and were conducted in a location determined by the participant (e.g., clinic, personal office, or the home of the family member of a palliative patient). Interview guidelines were derived from the conceptual framework for the evaluation of integrated palliative care networks [1], which covered the key topics to explore the perceptions of quality of care. Interview questions for professionals included questions about aspects of quality of care, such as satisfaction with care, access to palliative care, and perception about the client-centeredness. Questions about perceptions of satisfaction/access with care included: "Would you say that the services of your palliative care network are available/accessible to all the patients?" and "Are patients satisfied with the care they receive through your network?". On the other hand, perceptions of the client-centeredness included the following questions: "How satisfied are the patients with the communication and information transfer within the framework of palliative care?", "Do you have the impression that family/friends of patients are appropriately involved in the palliative care provided by your network?" and "Do you think that your network can adequately consider the needs of the patients? (medical, non-medical, and spiritual)" (Table 2). While the questions proposed to family members covered a range of questions gauging perceptions about the following aspects of care: the availability/access to care, client-centeredness of care, satisfaction with physical care (including pain and symptom management), and psychological care, as well as the transfer of communication and information. These questions included: "Were the palliative care services (previously) easily accessible to you and your relatives?", "How satisfied is your relative with the physical care that he/she has received?", "How satisfied is your family member with the psychological care that he/she receives?", "Do you feel that your family member is at the center of all decisions, activities, and efforts made by the palliative care team?", "When you think about the wishes, needs, and preferences of your relatives, do you think that they are 
respected by the palliative team?" and "Do you know about your family member's future treatment plan and are you satisfied with the plan?" (Table 3). Participants were encouraged to elaborate on the answers and provide relevant examples from their experience, wherever appropriate. Demographic data was collected from all the participants, and interviews were audio-recorded and transcribed verbatim in their original language, with all the personal identifiers being removed.

Table 2 Interview questions for palliative professionals

1. Would you say that the services of your palliative care network are available/accessible to all the patients?

2. Are patients satisfied with the care they receive through your network?

3. How satisfied are the patients with the transfer of communication and information within the framework of palliative care?

4. Do you have the impression that family/friends of patients are appropriately involved in palliative care provided by your network?

5. Do you think that your network can adequately consider the needs of patients? (medical, nonmedical, and spiritual)

Table 3 Interview questions for the relatives of palliative patients

1. Were the palliative care services (previously) easily accessible to you and your relatives?

2. In your opinion, how satisfied is your relative with the physical care that he/she received?

3. In your opinion, how satisfied is your family member with the psychological care that he/she receives?

4. Do you feel that your family member is at the center of all decisions, activities, and efforts of the Palliative Care team?

5. When you think about the wishes, needs, and preferences of your relatives, do you think that they are respected by the Palliative Care team?

6. Do you know about your family member's future treatment plan, and are you satisfied with the plan?

\subsection{Data Analysis}

The data were analyzed using the thematic analysis [22] to identify and report the patterns within. The first stage of the analysis involved familiarization of the data, i.e., listening to the audio 
recordings and reading through the transcripts. The second stage was coding the interview data. Firstly, the data was divided into the transcripts between the interviews of professionals and the ones with the relatives. Two researchers were initially involved in the coding of data, where one researcher coded the data of the interviews with professionals, and the other coded the data of the relatives. The first few transcripts from each dataset were manually coded by the researchers. But, since the transcripts were in different languages, the researchers had to aid one another according to their linguistic ability, where one researcher was fluent in French and German, the other was fluent in Italian. Thus, the researchers explained important segments of the data to one another in their commonly spoken language, English which was otherwise not understandable due to the language barrier. After this, the researchers consulted with one another to compare the discovered perspectives within the data related to the key topics (i.e., availability of care, the client centeredness of care, and satisfaction with care (including physical and psychosocial care), and communication and information transfer). The converging and diverging perspectives between the two interview groups were identified, continuously re-examined, and agreed upon between the researchers to ensure their consensus regarding the codes and analysis. The data in the subsequent transcripts were then coded according to the prominent perspective. Later, the data were coded using a qualitative data analysis software package (MAX QDA) to organize the data efficiently and systematically.

\subsection{Ethical Consideration}

The study received formal research approval from the Ethics Committee of Northwestern Switzerland (EKNZ) on the 29th of August 2018, (Req-2018-00490). Before commencing the interviews, participants received and completed an informed consent form for the study and recording of interviews and the researchers provided them the information about the objectives of the study and the reasons for recording the interviews. Moreover, the concepts of voluntary participation and confidentiality of data were explained to ensure their agreement in the participation of the study.

\section{Results}

The comparison between the perceptions of professionals and the relatives of palliative patients resulted in considerable differences in the following aspects of care: 1) the availability, 2) clientcenteredness (including respect for patient wishes and relatives involvement in care, 3) satisfaction (including physical and psychosocial), and 4) communication and information transfer. These perceptions are compared and described below in detail.

\subsection{Availability of Care}

The availability of care refers to the perception of services being accessible to those who need them, in time [23]. The results indicated considerable divergences in this crucial element of the quality of care, where professionals perceive that they are highly available to patients/relatives to provide palliative services, while relatives perceive that they are: a) poorly informed about the availability of care, b) access to care was a result of independent initiative rather than direction from professionals, and c) despite professionals insisting on the provision of all the relevant information 
about the available palliative services to patients/family members, relatives felt underprepared and also less aware about the palliative care services. These findings are further explained below.

\subsubsection{Perspectives of Professionals}

Professionals did not report problems with the availability of care. Instead, they believed palliative services were easily accessible to patients. According to GPs, patients were well informed about the services available to them. As one of the GPs explained: "they know what services are around and are given all the information they need. We tell them everything there is to know". Another GP supported this notion by adding that plenty of additional information was available for patients online and in hospitals or clinics regarding the care treatments. He quoted: "We have leaflets about services here, and all you have to do is type in "palliative.ch" and offers will come up".

\subsubsection{Perspectives of the Relatives}

In contrast to the professionals, relatives of palliative patients reported the following issues concerning the availability of care. Firstly, they perceive that only limited information about services is provided to them. Additionally, they reported that they must seek the information about services themselves.

3.1.2.1 Limited Provision of Information about Palliative Services. A high number of family members disagree on being informed about the services by professionals rather, they reported that they accessed palliative care services informally, i.e., by hearing about palliative care through friends or family: "It was more through word of mouth, i.e., the people who also had their relatives in palliative care, only told about it and enlightened us (about palliative care)". Moreover, relatives suggest that the degree of information and support provided may be overestimated by GPs, as relatives explained that patients are not always made aware of the services available to them. For example, the daughter of a palliative care patient explains: "They (doctor) said your mother needs something, but then where was it? I do not know whom to approach. Maybe even after a week, nobody gets in touch with me and in the meantime, I do not know where I am supposed to go with her (mother)". Relatives also describe that they were not prepared for palliative care services because it was not explained to them by the professionals. As one mother recalls: "I thought when they found a lump there, she (daughter) would be referred to a gynecologist. I did not know that she needed an oncologist, no one explained that to me. I was suddenly told, "oh, here is the oncologist", which made me realize that it must be cancer".

3.1.2.2 A 'Laissez-faire' Attitude in Providing Palliative Services. Many relatives indicated that the patients and relatives must take an initiative to find out the information about palliative services on their own. For instance, one relative says: "It is accessible and if you type "Palliative Care Luzern", then it comes to this palliative community via Google. I think it is very clear if you are a little interested and look it up yourself", while another respondent remarks: "So, you have to inquire and know where to inquire." Further, the responsibility to organize palliative services for family members are perceived to fall often in the hands of family members and is not facilitated enough by professional support. As one relative explains that the health care professionals did not provide enough practical support for accessing palliative care services: "Hospital did not help much. I needed 
a bed and a wheelchair and I had to organize everything by myself. She (nurse) just gave me the phone numbers." Similarly, another family carer argues that without professional help, it may not be so easy for an average person to find out about palliative care services because one may not be already familiar with the medical services and may struggle to get the information and access to palliative care services: "Because I work at the hospital, it is easier for me and accessible also but I do not know what it is for someone who is not that smart."

3.1.2.3 Limited Awareness of Palliative Care. In a few cases, it was also evident that relatives had limited awareness, not only about the services but palliative care in general. For instance, one family member describes how they were not informed about palliative services and were left to find it out themselves. As one relative describes: "I thought to myself, "But what is palliative care for? What the hell are they doing?" and they just said to me, "make a contact, and you will see". Similarly, a partner of the palliative patient describes that despite being treated in palliative care, her partner was not explained what palliative care services were for and thought that they were only relevant in the case of a terminal illness: "my partner had the feeling that palliative care comes into play only when there is nothing left to do and it comes down to dying". Similarly, another relative explained that she was not made aware of palliative care services until a much later stage of her family member being in care. She did not expect it to be relevant because she had only heard of it in the context of HIV AIDS: "It was only later that the doctor drew my attention toward the possibility of palliative care. Palliative medicine was too little known in my eyes, except in the case of AIDS."

\subsection{Client Centeredness of Care}

The key aspects of client centeredness in care include perceived respect for patient needs and preferences and also the involvement of family members in care, which is discussed below.

\subsubsection{Respect for Patient Wishes}

The results indicated a shared consensus between the professionals and family members of palliative patients about considering and respecting the needs and wishes of the patients during the care process. Notably, many professionals expressed their opinion on the patient being "the center" of all care decisions. As one specialist doctor describes: "Of course, it all depends on the client. We try to take into account all their desires and see them as a person, on the whole". In general, family members agreed that professionals respected the patients' wishes while treating them. One family member quoted an example of their son who was in the care and requested that his father should accompany him to all meetings with the GP that was ensured: "He did not feel confident being in those meetings on his own because it was often overwhelming, but when he asked that I attend and take notes for him, the team did everything to accommodate us. We then worked around a schedule that suited all of us". However, there were also some notable complaints from family members who felt that the patient was not truly at the "center" of care because the health care practitioners working with them were too busy to dedicate time to the patient, hence they did not feel like their family member was a priority. Although one mother acknowledged the inevitability of this situation. Admitting to her frustration, she quoted: "I, of course, accept that the world does not revolve around us and the nurses are busy. But it is not nice to feel that your daughter is just one of many patients because they just do not have time to talk to us about what she wants. They have 
too many other people's needs to think about". Similarly, another relative supported this notion about patients not being given the full attention of health care professionals. Instead, he thought they had to fight for it. Otherwise, they risk being forgotten: "He (the patient) does not draw attention to himself and is very reserved. He does not get attention at all. So, they just leave him alone. Someone who is always shouting gets a lot more attention".

\subsubsection{Relatives' Involvement in Care}

3.2.2.1 Perspectives of the Relatives. A vast majority of relatives reported feeling intensely involved in the care process. Many of them describe playing a crucial role in the care of their loved ones in the treatment plan and decision making. For example, one relative explains the fundamental role in the decision-making process toward the care of the patient: "There was no decision made without her and my consent. We were completely in agreement and involved in the decision-making and reflection process." Similarly, many others also quoted examples of knowing the treatment plan and schedule of their relatives' care to support their involvement and often described themselves as a part of the care team. As one daughter explains: "I have a black file here filled with every detail about when and which medications she should take. I know everything at this point. The nurses leave me to tend to her bandages and so on because they know I am no different to them, I am also one of her carers". However, in two cases, the family members reported that they did not feel involved enough in the care process, not because the professionals failed to keep them involved, but because of the resistance from the patient. As explained by a mother: "He (son) refuses to let me in the meetings. He wants his treatment to be between him and his doctors, and I cannot override that, unfortunately, I am just on the sidelines".

3.2.2.2 Perspectives of the Professionals. Most professionals agree that family members are fully involved in the care. As described by one nurse: "I know mothers and daughters who could tell you more about the pain medications of their relative than the nurses on the same team who are there for years. They are absolutely involved!". However, in some cases, professionals felt that relatives were too involved. For instance, one GP feared that the relative risked 'burn out' from the extent of his involvement with care: "I saw the husband doing everything he could, he invested everything at the cost of his career, his training. It was incredible and there was little compensation. There was no tax allowance to support this. I have to be very careful about the burn-out of the spouse who could not take it anymore. The spouse can sometimes get out of control, and then psychologically, it becomes difficult.". This is in line with a specialist doctor who admitted to not include the family members much because they are too emotionally invested. The specialist feels wary of involving relatives too intensely in the decision-making process: "When it comes to making decisions, unfortunately, they cannot be trusted to make the 'best' decisions, let's say because their emotions are in it too". However, despite family members feeling extremely involved in the care process, responses from some GPs suggest that the family members may not be as involved as they might think. Another doctor admits that he with-holds the information from relatives in some cases: "I do not want to gloss over this, of course, conversations also take place behind the patient's back, or it is expressed that one should not communicate certain things with the patient, which is quite difficult for me, it is legally a gray area". Similarly, a few GPs mentioned difficulties in involving the families for practical reasons such as not all family members agree on a course of action related to the care. As one doctor describes: "Families are always involved but it is not always easy with them, because 
you have a son from Geneva who does not agree with the son from Lausanne. The son from Lausanne thinks that you should not put her (mother) in a palliative care center and she should die at home while the one from Geneva thinks it is better to have care."

\subsection{Satisfaction with Care}

Satisfaction with care describes the perception of how satisfied professionals and family members think the patients to be with their overall care, including physical and psychosocial care. The perspectives are further described below.

\subsubsection{Perspectives of the Professionals}

The results demonstrated that both the professionals and family members perceived the patients to be satisfied with the care. However, professionals often acknowledged their limited capacity in estimating the satisfaction of patients. When asked by a family doctor whether he believed his patients to be satisfied with the care they received, he said: "I do not know. How do we evaluate that? (Should we ask) Was it all right? How are you doing? Most of the people I sent to a mobile unit or hospital felt satisfied. But it is only an impression". Yet, on the whole, professionals generally believe that patients were satisfied. One nurse even goes as far as to say: "I do not think I have ever known a patient to be truly unhappy with the care they get here".

\subsubsection{Perspectives of the Relatives}

Although the majority of family members seem to be happy with the care and feel that their family members were content with the received palliative care, many family members also raised some specific issues negatively impacting their perception about satisfaction with care. Notably, relatives often attributed the dissatisfaction with overall care to a) poor coordination, b) a perceived lack of professional competency, and c) poor continuity of care. Moreover, family members also had complaints about physical and psychosocial care, especially a lack of psychosocial support. The aspects of care to perceived satisfaction are expanded below.

3.3.2.1 Poor Coordination of Care. The relatives commonly expressed that palliative care teams were poorly coordinated. For instance, a relative describes that her mother's care was compromised due to the poor coordination of care and time: "It was badly organized. The nurses never came on time. They also had no plans about when to come. It was agreed at about 8 o'clock, but by the time they came, it would be 10 o'clock. In the meantime, my mother was lying on the bed with wet diapers and could not cope anymore". Similarly, one person explains a situation where the care was badly coordinated because professionals were not sure of the medications being already received by the patient: "They were not well informed and did not appear to know what was going on. They did not even know if they would give him any medication. When we saw that they were not sure, I was not happy". Additionally, some relatives mentioned their unhappiness with the care because the coordination was unreliable. One relative describes their frustration: "Depending on the time, there were very quick changes happening, and every time there was someone else. So, consistency was often missing, actually continuously (missing)". Similarly, another relative pointed out that they could not answer the question about how satisfied they were with the care because of the 
unpredictability of the level of care: "One day you might get the best team ever and it is all great, but just as quickly, you find yourself with strangers on the team, and it is just the opposite of great!"

3.3.2.2 Poor Continuity of Care. Continuity of care is an important aspect that refers to the relational continuity (the patient is continued with the usual practitioner), informational continuity (continued communication and knowledge for easy flow of patient information between the involved care providers), and management continuity (coordination for transitioning between care providers to be clear and seamless for the patient) [24]. Some family members describe their frustration: "I cannot do anything for you anymore (said by a professional). That is another shock you can imagine". Understandably, family members also reported suffering because of the sudden drop in communication with professionals after they no longer needed their care or were transferred to another care facility. As one relative describes, there was no form of continuity of care once the treatment for the patient ended: "From one day to the next, I went from seeing their (nurses) faces any time I needed them, to suddenly being all alone again. I think that is a terrible part of care because my life goes on, but the care just stops". Yet another person explains a situation where there was no continuity of care after the death of their partner and they were left without knowledge about the next steps: "When my partner died, the only thing I thought was, now I have to call somebody, and I do not know who, I do not know what to do now."

3.3.2.3 Lack of Professional Competency. Alarmingly, quite a few family members reported dissatisfaction with the quality of care because they felt that the professionals were incompetent. As one person puts it bluntly: "I was not happy with the home care service because they may be nice people but they are not competent". Similarly, another relative was unhappy with the care and wanted to replace the professional because she felt that the professional was not competent: "Let's just say, I had to deal with a nurse who was not up to the task. I talked to the head nurse about it and said, "This one (nurse) has to get out of here. It is not okay at all". Another relative speculates that the standard of care could be lower now because of the lack of sufficient training: "The level of care has gone down. I have the impression that it has dropped because some foreign doctors do not have the level of Swiss studies (education)". In another situation, the relative implies that the competency was so poor that they liked to step in themselves to carry out the treatments: "I liked to be a volunteer for a while because I noticed that some treatments were sometimes poorly done". She goes on to explain that the nurses failed to meet the needs of the patient and were not attended in times of need: "For example, they did not give her anything to drink for a very long time and she was dying of thirst after she was cold, and then she was hot because of her changing temperature but no one passed in to help."

\subsubsection{Physical and Psychosocial Care}

Physical care involves medical and practical aspects along with pain and symptom management [25] while the psychosocial care involves meeting the emotional, psychological, and existential needs of palliative patients and their families, and help in alleviating grief, fear, and other psychological and social problems [1]. The results indicated the following key perspectives: a) psychosocial care was lacking, b) physical care was prioritized over psychosocial care, and c) relatives felt conflicted about the approaches to pain and symptom management. The data on these perspectives are discussed below. 
3.3.3.1 Lack in Psychosocial Care. Professionals support that many services are available for treating both physical and psychological needs, and doctors and nurses do attend to these demands of patients and their relatives. As one adamantly puts: "It is not just a job, but it is my duty to make sure that they (patients) are looked after in every way imaginable. This is palliative care; it is about giving them the best possible life at this stage". Despite this, both family members and professionals seemed to agree that psychosocial care is considerably lacking compared to physical care. One GP expresses that they are not comfortable providing psychological support compared to the physical support and admit to not being concerned with the 'spiritual' needs: "Every day, medical needs I can manage, but not spiritual needs. We are not very good at this. I am rarely interested in knowing what the patient's spiritual position is. I am rather involved in all kinds of things about the pain but I am not very good at this psychological thing". Moreover, another GP implies that although psychological issues should be addressed, it is difficult to provide the support because these problems are expected and are considered 'normal' in palliative care: "Spiritual needs should not be neglected, nor depression, but when you realize, "She has cancer", it is normal that she is sad". What should we do about her depression?" This may account for why professionals also report that they do not necessarily advertise the options for psychosocial support but rather only provide it when requested. As one GP explains: "There are psychologists and support groups available, especially for bereaved widows but I give this information only when asked. I do not usually ask them (patients) about their spiritual needs".

3.3.3.2 Physical Care Prioritized Over Psychosocial Care. Perhaps, such thinking from the professionals might account for many family members also reporting their relatives' psychological needs not being taken as seriously as the physical ones. One concerning example was provided by a relative who describes that their relative received proper care only when she started threatening to take her own life: "She wanted to get it over with and she said, "I am going to get a knife and kill myself" and then I went to the doctor and said, "Now you listen to what she said to me and do something" and then they said, "OK, we heard her despair". Oh, yes only then it was more physical, that is what they meant by 'despair'. We had to wait a long time because I felt the despair long before that". Also, family members admit having limited knowledge about where to receive the psychological help, as one father describes: "I have heard of churches sending people out to come and talk with you. But I am not sure that this is something people like us would have the chance to do".

3.3.3.3 Relatives Feeling Conflicted about Pain and Symptom Management. On the other hand, some family members reported feeling conflicted about physical care, including pain and symptom management (i.e., commonly, the painkillers or sedatives, e.g., morphine). Although the majority of relatives desired their family members in care to be in as little pain as possible, they also struggled with the perceived negative impact on the patients' quality of life. As one relative describes the adverse effects of pain killers: "Well, we try to relieve the symptoms, and calm the pain, but the more we subside the pain, the more the person's personality goes away, which I find difficult to bear. She (family member) is concerned that she would "end up like a vegetable" and that decision would be made in her place." Another mother describes a similar concern: "If we say, yes, up the dosage, then the doctor will do so, but at what cost? Perhaps her life will be spared, but what kind of life are you saving? She is totally out of it, on medications". 


\subsection{Satisfaction with Care}

Communication and information transfer refer to all the aspects of the provider's collaboration and patient-centered interactions [1]. The sharing of information that is relevant, accurate, transparent, concise, and timely is an essential element to ensure a high quality of palliative care [26]. Responses from professionals and relatives echoed similar concerns for communication between the relatives, patients, and professionals and the transfer of important care information. Crucially, the majority of professionals reported issues with finding time to talk with patients or their relatives. As one assistant nurse explains: "It goes without saying that we are all busy around here. I do my best, but I am limited to quick pop-ins". Another GP admitted that despite trying to reassure the patients that he is available for them, he often misses the phone calls and cannot attend meetings: "I tell them I am reachable 24/7 which is technically true but practically not. My days are so full, one visit runs over and then I cannot make the next one". Also, some family members reported that information about patient care was poorly explained or lacking, as one relative describes: "They (nurses) explained it badly to us. They should have said when she is unconscious or she sleeps then you should not give her anything to drink, but they did not tell us that." In other cases, relatives describe miscommunications because the information was not passed on to them directly. One family member describes that they waited a long time for nothing because a message was relayed to them: "She (nurse) should have come but had an emergency and could not come. She reported it to the central office, but then they forgot to pass on the message to us and we waited two hours after all that."

\section{Discussion}

The findings of this study highlighted important aspects of the quality of palliative care. Specifically, in indicating the valuable perspectives and experiences of the professionals and relatives of palliative patients regarding the availability, client centeredness, satisfaction with care, and communication and information transfer.

Firstly, the data reveals a high level of disagreement between professionals and relatives' opinions toward the availability of care. Crucially, although professionals perceived that palliative services were highly available to patients and their relatives, relatives expressed being poorly informed about it. Instead, they felt 'left alone' to discover palliative services for themselves, or when encouraged to seek palliative services, the responsibility to take the initiative was on them. It was also apparent that professionals mainly understood care to be available to patients because of the presence of infrastructure for services. However, relatives described that since professionals did not inform patients about these services, it was not easily available or accessible. The experiences of relatives describing the situations where professionals do not adequately inform patients about the availability of care are highly concerning because failing to provide patients with care information could result in missing out on the appropriate treatment, which at the palliative level may contribute to further deteriorating health outcomes to already fragile patients. A vast body of literature supports that misinformation can also negatively impact the quality of patient care because the coordination of care is prohibited by inaccurate or untimely information [27]. If the patients who require specialized care in palliative services are not made aware of or connected to these services, then they may risk neglecting their health at a potentially critical point of their illness trajectory [1]. If this is the case, as the relatives imply, where patients and relatives are not 
sufficiently informed about the services available to them and/or they must rely on themselves to reach out to services, then it not only indicates a considerable failing on behalf of professionals to meet the patient needs but also clearly demands them to re-evaluate their approaches in providing the service information.

Another important finding is the divergent perceptions of the client centeredness, especially the involvement of relatives in care. Adequate involvement of relatives in care is a vital aspect of the quality of care [9]. Yet, the responses indicated that while the relatives and professionals agreed on relatives being highly involved in care, they differed in the opinions for appropriateness of their involvement. Specifically, health care professionals often expressed concern with the extent of involvement of relatives in the process of care. Many explained that they were worried about the family members taking too much responsibility for their relatives' care and risked psychological distress, including 'burn-out'. Consequently, a few GPs alluded to concealing information from the family members regarding the patient, out of the fear of causing too much stress, particularly during decision-making. The concerns expressed by the GPs here are legitimate, especially because extensive literature supports the association between the caregivers and a high risk for a whole host of adverse psychological effects, including, but not limited to depression, anxiety, burn- out, stress, fatigue, and insomnia $[28,29]$. The conflict expressed by the GPs here needs the relatives to be adequately involved in care but also raises the question about the extent of their involvement in care, or, in what capacity? There is a growing body of evidence to support that relatives are not only involved in care but are also becoming an increasingly fundamental part of the palliative care team [30]. In many cases, family members are described as 'primary caregivers' in palliative care literature, i.e., individuals who provide most of the care to the patients without payment [12]. While other countries, including the United Kingdom and the United States, have adopted strategies to financially support such primary caregivers [31], Switzerland does not yet support the health regulations enabling such initiatives to a large extent. Relatives living in the same household may receive some financial reimbursement, but that does not apply to all cases, especially those who care for the patients living separately from them [32]. This may also contribute to the burden that health care professionals have described in the data. Adequate reimbursement in health care is of utmost importance to ensure good quality care and alleviate stress for carers [33]. Beyond that, appropriate training and emotional support should be provided to equip the relatives to manage the high level of involvement while taking care of their loved ones.

The concerns raised by the relatives of palliative patients related to the satisfaction of care are important discoveries made in this study because health care professionals in the field often felt that they could not necessarily comment on the satisfaction of patients. The relatives were able to shed light on the specific issues that negatively impacted their evaluation of care, they also pointed out several factors, such as poor coordination, poor continuity, as well as negligence of psychosocial needs, and a perceived lack of professional competency. Poor care coordination is consistently identified as a problematic facet of care in the context of palliative services [34]. These results echo recent literature concerning the coordination of palliative care services in Switzerland highlighting some of the problems described here, such as the poor organization of care resulting from frequent changes happening in the care team, miscommunication, and a lack of time for care coordination [17]. They further advocated the need for improvement in this regard. Regarding the perceived poor continuity of care, in context to the problems with communication and information transfer, findings indicated a need for strengthened communication between the patients, relatives, and 
providers, and one way of aligning this is through the allocated care coordinators who facilitate the care coordination and communication between the members of the palliative team [20]. Notably, palliative care settings with methods such as the employment of case managers who manage the smooth transitioning of patient care continuity have been well received by the patients and relatives [35].

\section{Conclusion}

This study brought different perspectives toward the quality of palliative care to light. Significantly, professionals working in the field and relatives of palliative patients held different beliefs toward the key aspects of care, including the availability and access to care, client centeredness of care, satisfaction with care (especially in case of psychosocial care), and communication and information transfer. Although there was a consensus between the professionals and family members for various dimensions of care, this study highlighted important apparent divergences between professionals and relatives' perspectives. Significantly, the interviews of the family members showed that though the health care professionals strive to provide the highest quality of care, there are some aspects where it lacks. The primary concerns were that care was not always readily available, poorly coordinated, patient needs were overlooked because of the lack of time, few professionals were perceived to be incompetent, communication was lacking, and psychosocial needs were neglected. Professionals agreed that psychosocial needs are not well addressed as the physical needs and also reported communication problems. However, professionals also raised other concerns with aspects of care, where they felt that though it is good for relatives to be involved in care, their over-involvement may lead to a high risk of burn out caused by the high stress and emotionally taxing circumstances of palliative care. These different perspectives, including both similarities and discrepancies, represent a demand for addressing the shortcomings of palliative care services.

\section{Recommendations}

In response to the findings of the study, the following propositions are suggested for the health policies of palliative care in Switzerland. Firstly, because of the significant involvement of family members of palliative patients in the process of care, adequate psychosocial and financial support should not only be available but mandatorily provided in the case of family members being primary caregivers. This measure is proposed to alleviate the psychological and financial burden from relatives and reduce the risk of adverse health and financial outcomes often caused by being involved in palliative care. Additionally, professionals in the field are urged to provide relevant information about the availability of basic palliative care along with psychosocial support. The education of palliative professionals should ensure that they are equally equipped to cater to the psychological issues as they are to physical ones to avoid the negligence of psychosocial needs. For the coordination issues faced in palliative care teams, designated care coordinators should be employed to mitigate the miscommunication and untimely delivery of information. 


\section{Limitations}

This study relies on the perspectives of professionals and family members. However, it would be invaluable to have palliative patients' insight and perceptions for understanding the patients' experience of the quality of care in palliative services. This information could serve important knowledge to inform the policy and provide recommendations concerning the quality of care in palliative services with patient wishes in mind. Another drawback of the study, as acknowledged by some of the interviewed health care professionals, is that it is difficult to accurately assess the quality of care without having measurable scales, and it is uncertain to what extent professionals and relatives can evaluate the standard of care since they are not the patient themselves. Specifically, many studies support that health care professionals tend to overestimate the patients' satisfaction and overall quality of care provision [36]. Although it is recognized that health care professionals and relatives of patients may have an excellent insight into the patients' experience of care, the most accurate understanding of the quality of a patient's care would inevitably be the one directly coming from the patient.

\section{Acknowledgments}

The authors wish to thank all the participants of the study for kindly contributing their expert knowledge and insights of palliative care, which were crucial to explore the quality of palliative care. The authors also wish to thank the Swiss National Science Foundation (SNF), NRP 74 "Smarter Health Care" (www.nrp74.ch; Schweizerischer Nationalfonds zur Förderung der Wissenschaftlichen Forschung) for funding this research.

\section{Author Contributions}

Ms. Emily Reeves is the corresponding author of this work. Ms. Emily Reeves and Dr. Prof. Brigitte Liebig were responsible for the conceptual development of this work. Additionally, Ms. Emily Reeves carried out the write up of this research, whilst Dr. Prof. Brigitte Liebig expertly edited and refined the paper. Ms. Emily Reeves and Eveline Degen-Jermann carried out the qualitative interview studies and were responsible for the qualitative analysis of the interview data. The share of labour with respect to the analysis of the interviews was divided equally between Ms. Eveline Degen and Ms. Emily Reeves.

\section{Competing Interests}

The authors have declared that no competing interests exist.

\section{References}

1. Bainbridge D, Brazil K, Krueger P, Ploeg J, Taniguchi A. A proposed systems approach to the evaluation of integrated palliative care. BMC Palliat Care. 2010; 9: 8.

2. Hanson LC, Bull J, Wessell K, Massie L, Bennett RE, Kutner JS, et al. Strategies to support recruitment of patients with life-limiting illness for research: The palliative care research cooperative group. J Pain Symptom Manag. 2014; 48: 1021-1030. 
3. Darzi A. High quality care for all: NHS next stage review final report. London: Department of health; 2008. Available from: https://www.gov.uk/government/publications/high-quality-carefor-all-nhs-next-stage-review-final-report.

4. High quality-safe services-quality and patient safety in the health and care services. Report to the storting (norwegian parliament) No. 10. Olso: Ministry of Health and Care Services; 20122013. Available from: https://www.regjeringen.no/no/dokumenter/meld-st-1020122013/id709025.

5. Wittenberg-Lyles E, Parker OD, Demiris G, Baldwin P, Regehr K. Communication dynamics in hospice teams: Understanding the role of the chaplain in interdisciplinary team collaboration. J Palliat Med. 2009; 11: 1330-1335.

6. Bengoa R, Kawar R, Key P, Leatherman S, Massoud R, Sturno P. Quality of care: A process for making strategic choices in health systems. Geneva: World Health Organization (WHO); 2006. Available from: http://www.who.int/iris/handle/10665/43470.

7. What is quality of care and why is it important? Geneva: World Health Organization (WHO); 2020. Available from: https://www.who.int/maternal_child_adolescent/topics/quality-ofcare/definition/en.

8. Austin L, Luker K, Caress A, Hallett C. Palliative care: Community nurses' perceptions of quality. Qual Health Care. 2000; 9: 151-158.

9. Burns CM, Abernethy AP, Grande DE, David CC. Uncovering an invisible network of direct caregivers at the end of life: A population study. Palliat Med. 2013; 27: 608-615.

10. Gomes B, Calanzani N, Higginson I. Reversal of the British trends in place of death: Time series analysis 2004-2010. Palliat Med. 2012; 26: 102-107.

11. Hauser JM, Kramer BJ. Family caregivers in palliative care. Clin Geriatr Med. 2004; 20: 671-688.

12. Kitrungrote $L$, Cohen MZ. Quality of life of family caregivers of patients with cancer: A literature review. Oncol Nurs Forum. 2006; 33: 625-632.

13. Aasb $\varnothing$ G, Rugkåsa J, Solbraeække KN, Werner A. Negotiating the care-giving role: Family members' experience during critical exacerbation of COPD in Norway. Health Soc Care Community. 2007; 25: 612-620.

14. Brobäck G, Berterö $C$. How next of kin experience palliative care of relatives at home. Eur J Cancer Care. 2003; 12: 339-346.

15. Oosterveld-Vlug MG, Custers B, Hofstede J, Donker GA, Rijken PM, Korevaar JC, et al. What are essential elements of high-quality palliative care at home? An interview study among patients and relatives faced with advanced cancer. BMC Palliat Care. 2019; 18: 96.

16. Ke YX, Hu SH, Takemura N, Lin CC. Perceived quality of palliative care in intensive care units among doctors and nurses in Taiwan. Int J Qual Health Care. 2019; 31: 741-747.

17. Vedel I, Ghadi V, Lapointe L, Routelous C, Aegerter P, Guirimand F. Patients', family caregivers', and professionals' perspectives on quality of palliative care: A qualitative study. Palliat Med. 2006; 28: 1128-1138.

18. Brazil K, Bainbridge D, Ploeg J, Krueger P, Taniguchi A, Marshall D. Family caregiver views on patient-centred care at the end of life. Scand J Caring Sci. 2011; 26: 513-518.

19. Hudson P, Payne S. Family Caregivers and palliative care: Current status and agenda for the future. J Palliat Med. 2012; 14: 864-869. 
20. Reeves $E$, Schweighoffer R, Liebig B. An investigation of the challenges to coordination at the interface of primary and specialized palliative care services in Switzerland: A qualitative interview study. J Interprof Care. 2020; 26: 1-7.

21. Alvarado $\mathrm{V}$, Liebig B. Inter-professional collaboration between family doctors and nurses at the end of life. Challenges of community-based palliative care in Switzerland. J Comm Pub Health. 2016; 2: 124.

22. Braun V, Clarke V. Using thematic analysis in psychology. Qual Res Psychol. 2006; 3: 77-101.

23. Shaller D, Consulting S. Patient-centered care: What does it take? New York: The commonwealth fund; 2007. Available from: https://www.researchgate.net/publication/228787332_Patient_Centered_Care_What_Does_ It_Take.

24. Reid Rj, Haggerty J, Mckendry. Defusing the confusion: Concepts and measures of continuity of health care. Final Report. Ottawa: Canadian Health Service Research Foundation; 2002. Available

from: https://www.researchgate.net/publication/245856177_Defusing_the_Confusion_Concepts_a nd_Measures_of_Continuity_of_Health_Care.

25. Ferris FD, Von Gunten CF, Emanuel LL. Ensuring competency in end-of-life care: Controlling symptoms. BMC Palliat Care. 2002; 1: 5.

26. Oandasan I, Baker GR, Barker K, Bosco D, D'amour D, Jones S, et al. Teamwork in healthcare: Promoting effective teamwork in healthcare in Canada Ottawa. Ottawa: Canadian Health Services Research Foundation; 2006. Available from: https://www.researchgate.net/publication/249940003_Teamwork_in_Healthcare_Promoting _Effective_Teamwork_in_Healthcare_in_Canada.

27. Luckett T, Phillips J, Agar M, Virdun C, Green A, Davidson P. Elements of effective palliative care models: A rapid review. BMC Health Serv Res. 2014; 14: 136.

28. Sand L, Strang P, Milberg A. Dying cancer patients experiences of powerlessness and helplessness. Support Care Cancer. 2008; 16: 853-862.

29. Totman J, Pistrang N, Smith S, Hennessey S, Martin J. You only have one chance to get it right: A qualitative study of relatives experiences of caring at home for a family member with terminal cancer. Palliat Med. 2015; 29: 496-507.

30. Woodman C, Baillie J, Sivell S. The preferences and perspectives of family caregivers towards place of care for their relatives at the end-of-life. A systematic review and thematic synthesis of the qualitative evidence. BMJ Support Palliat Care. 2016; 6: 418-429.

31. Gardiner C. Exploring the financial impact of caring for family members receiving palliative and end-of-life care: A systematic review of the literature. Palliat Med. 2014; 28: 375-390.

32. Nationale strategie (National strategy) palliative care 2013-2015. Bern: Federal Office of Public Health $\quad$ (FOPH); $2012 . \quad$ Available from: https://www.pallnetz.ch/cm_document/07_D_Nationale_Strategie_Palliative_Care_20132015.pdf.

33. Travis $S$, Hunt P. Supportive and palliative care networks: A new model for integrated care. Int J Palliat Nurs. 2001; 7: 501-504.

34. Reeves S, Xyrichis A, Zwarenstein M. Teamwork, collaboration, coordination, and networking: Why we need to distinguish between different types of interprofessional practice. J Interprof Care. 2018; 32: 1-3. 
35. Ozcelik H, Fadiloglu C, Karabulut B, Uya M. Examining the effect of the case management model on patient results in the palliative care of patients with cancer. Am J Hosp Palliat Med. 2014; 31: 655-664.

36. Hoffmann TC, Del Mar C. Clinicians' expectations of the benefits and harms of treatments, screening, and tests: A systematic review. JAMA Intern Med. 2017; 177: 407-419.

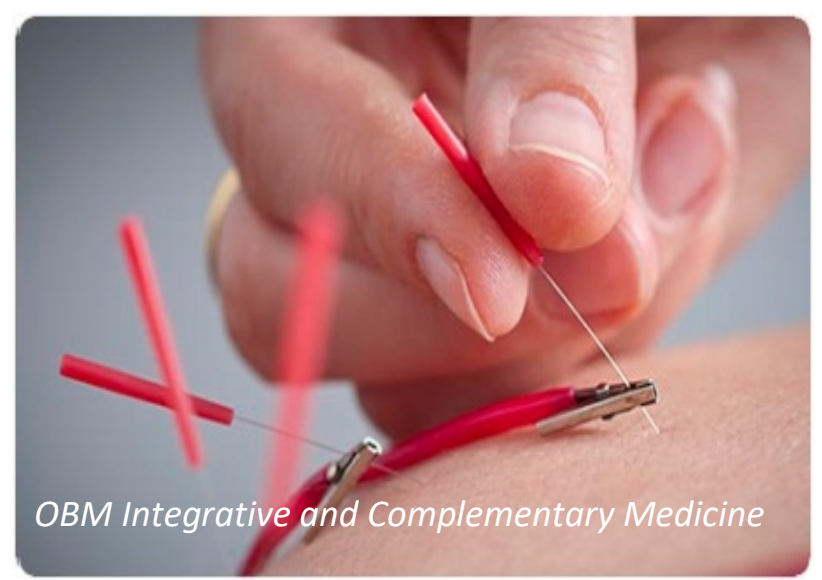

Enjoy OBM Integrative and Complementary Medicine by:

1. Submitting a manuscript

2. Joining in volunteer reviewer bank

3. Joining Editorial Board

4. Guest editing a special issue

For more details, please visit:

http://www.lidsen.com/journals/icm 